\title{
Variability of Platelet Reactivity on Antiplatelet Therapy in Neurointervention Procedure
}

\author{
Ho Jun Yi, M.D., Gyojun Hwang, M.D., Ph.D., Byoung Hun Lee, M.D. \\ Department of Neurosurgery, Hangang Sacred Heart Hospital, Hallym University College of Medicine, Seoul, Korea
}

As more intracranial aneurysms and other cerebrovascular pathologies are treated with neurointervention procedure, thromboembolic events that frequently lead to serious neurological deficit or fatal outcomes are increasing. In order to prevent the thromboembolic events, antiplatelet therapy is used in most procedures including coil embolization, stenting, and flow diversion. However, because of variable individual pharmacodynamics responses to antiplatelet drugs, especially clopidogrel, it is difficult for clinicians to select the adequate antiplatelet regimen and its optimal dose. This article reviews the neurointervention literature related to antiplatelet therapy and suggests a strategy for tailoring antiplatelet therapy in individual patients undergoing neurointervention based on the results of platelet function testing.

Key Words : Aspirin · Clopidogrel · Platelet aggregation inhibitors · Platelet function tests.

\section{INTRODUCTION}

Nowadays, neurointervention is increasingly being performed for the treatment of various cerebrovascular pathologies. However, because of the inherent limitation of the neurointervention procedure which is performed in the vessel, thromboembolic events which can lead to serious neurological deficits, are also increasing especially during the periprocedural period $^{39)}$. To prevent thromboembolic events, anticoagulation is being used as a tradition of endovascular treatment for a long time. Heparin can inhibit coagulation system immediately and effectively. In addition to anticoagulation, antiplatelet therapy is also used as a standard measure to further reduce thromboembolic events. However, antiplatelet therapy was found to have individual variability in pharmacodynamic response. Particularly, the variability of platelet reactivity on antiplatelet therapy is now recognized as the main risk factor for serious vascular events ${ }^{3,45}$.

The effect of heparin can be instantly measured, and thus easily controlled within proper therapeutic levels. To reduce the treatment failure of antiplatelet therapy, the effect of antiplatelet therapy also needs to be measured and controlled. Coronary intervention field initially accepted this approach and has published sufficient data to produce antiplatelet guidelines. Recently, this issue was also evaluated in many neurointervention studies including several prospective trials. Herein, we summarize these study data and suggest a strategy for tailoring antiplatelet therapy to individual patients under-

- Received : July 10, 2018 • Accepted : August 1, 2018

- Address for reprints : Gyojun Hwang, M.D., Ph.D.

Department of Neurosurgery, Hangang Sacred Heart Hospital, Hallym University College of Medicine, 12 Beodeunaru-ro 7-gil, Yeongdeungpo-gu, Seoul 07247, Korea Tel : +82-2-2639-5455, Fax : +82-2-2633-7571, E-mail : storynlemon@gmail.com

This is an Open Access article distributed under the terms of the Creative Commons Attribution Non-Commercial License (http://creativecommons.org/licenses/by-nc/4.0) which permits unrestricted non-commercial use, distribution, and reproduction in any medium, provided the original work is properly cited. 
going neurointervention.

\section{ASPIRIN RESPONSE VARIABILITY}

Aspirin inhibits platelet thromboxane A2 formation from arachidonic acid by irreversible acetylation of platelet cyclooxygenase 1 (COX-1). The term "aspirin resistance" generally refers to the situation in which aspirin is unable to acetylate COX-1 ${ }^{6}$. It may result from reduced absorption and bioavailability, in vivo inactivation, negative interactions with other substances, or polymorphism in COX-1. However, "true" aspirin resistance is known to be rare. In studies using a novel reference method that directly detects the acetylation or the lack of acetylation of platelet COX-1 by Western blotting, no patients was found with "true" aspirin resistance in healthy volunteers receiving low dose aspirin ${ }^{28,29)}$.

High platelet reactivity on aspirin therapy does not reflect true aspirin resistance but describes a status of platelet hyperreactivity while on aspirin therapy. COX-1 inhibition by aspirin affects only one of several pathways of platelet activation, and platelets with COX-1 inhibition can still be activated by other pathways. Therefore, routinely used platelet function tests show high inter- and intra-individual variability and considerable discrepancy is observed among results obtained by different tests. Furthermore, many clinical studies revealed no definite association between high on aspirin platelet reactivity and clinical outcomes. Currently, the cardiology guideline does not recommend changes in aspirin therapy based on laboratory test results ${ }^{2,5,6,11,32,38)}$.

In the neurointervention field, high on aspirin platelet reactivity was evaluated in several studies including one prospective trial. In these studies, high platelet reactivity was observed in less than $15 \%$ of the patients receiving aspirin before neurointervention $^{12,14,21,30,33,35,40)}$. However, thromboembolic events were found to be unrelated to high platelet reactivity on aspirin therapy. Therefore, there is no evidence supporting platelet function testing for aspirin response and changes in aspirin therapy based on their results in the neurointervention procedure.

\section{CLOPIDOGREL RESPONSE VARIABILITY}

Clopidogrel inhibits platelet aggregation by irreversibly binding to the P2Y12 subtype of the adenosine diphosphate receptor. It is an inactive prodrug that requires a complex enteric and hepatic metabolism. After enteric absorption, the majority of clopidogrel (up to 85\%) is metabolized in the blood by esterase- 1 to an inactive metabolite. The only remaining $15 \%$ are converted to active metabolite by two sequential oxidation steps by the cytochrome P450 (CYP) enzymes. CYP2C19 has the most prominent role and is involved in both steps.

Numerous studies have revealed that the antiplatelet effect of clopidogrel varies considerably among patients. CYP enzymes are a large highly polymorphic family of mono-oxygenases. Among them, loss-of-function variants in the hepatic CYP2C19 significantly alter the metabolism of clopidogrel and resultant drug effect. Ethnic difference is also observed, whereby the proportion of patients with poor metabolism is reported to be less than $25 \%$ of all clopidogrel-treated patients in the Western population, but is up to $40-50 \%$ in the Asian population ${ }^{37,41)}$. Drug-drug interactions also accounts for variability in response to clopidogrel. Especially, drugs related to CYP2C19 or CYP3A4 metabolism including calcium channel blockers, proton pump inhibitors, phenprocoumon, ketoconazole, antidepressants, and barbiturates may affect clopidogrel efficacy $^{13,42)}$.

Clopidogrel response can be phenotypically measured using the degree of platelet inhibition by laboratory tests. Platelet function test systems that are commercially available quantify the antiplatelet effect of clopidogrel by the use of platelet aggregometry, flow cytometry, shear-dependent assays, platelet counting, or other related methods such as thrombelastography. Traditionally, light transmittance aggregometry (LTA) has been used as the gold standard method. However, this method is not suitable for routine clinical examination, because it is time-consuming and requires intensive sample preparation. Moreover, its results are not instantly available. Therefore, the so-called "point-of-care" platelet function assays that provide rapid information for clinicians have been developed. The VerifyNow (Accumetrics, San Diego, CA, USA) is one of the most widely used point-of-care platelet function test systems. It has many advantages including full automation, use of a similar testing concept with LTA, good 
reproducibility, bedside use, and small blood volume requirement ${ }^{15,16,36)}$. More importantly, it has good correlation with LTA results ${ }^{15)}$. Clopidogrel response is reported with P2Y12 reaction units (PRU) on the VerifyNow system (Accumetrics) and categorized into high platelet reactivity (HPR; clopidogrel hypo-response), optimal platelet reactivity (OPR), or low platelet reactivity (LPR; clopidogrel hyper-response) according to the PRU cut-off value for each category. While \% inhibition was previously provided as an additional testing result, manufacture currently does not recommend its use, because of the lack of association between $\%$ inhibition and clinical outcomes.

\section{VARIABILITY OF PLATELET REACTIVITY ON CLOPIDOGREL THERAPY AND CLINICAL EVENTS IN NEUROINTERVENTION PROCEDURE}

The impact of variable platelet reactivity on clopidogrel therapy in patients undergoing neurovascular intervention has been analyzed in numerous studies. However, accurate comparison between the results of these studies is difficult, because of the variety of procedures, drug doses, study periods, and cut-off values for platelet reactivity.

For thromboembolic events in coiling or stenting, some retrospective studies showed no significant difference between patients with and without HPR on clopidogrel therapy ${ }^{27,34)}$. However, the majority of studies found an apparent association of HPR with thromboembolic events. Kang et al. ${ }^{22)}$ reported that high PRU value showed a significant tendency toward thromboembolic events ( $<240$ PRU, 4.3\%; 240-284 PRU, 2.2\%; 285-332 PRU, 6.5\%; >332 PRU, 17.0\%; $p=0.013$ ). Fifi et al. ${ }^{14)}$ found that HPR on clopidogrel therapy more frequently led to stent thrombosis in coiling or carotid stenting ( $16.7 \%$ vs. $1.6 \% ; p<0.01)$. Some authors evaluated high intensity lesions on diffusion weighted imaging (DWI) as a surrogate marker for thromboembolic events after the neurointervention procedure. Kim et al. ${ }^{24)}$ reported that HPR on clopidogrel therapy had a significant correlation with the high intensity lesions on DWI after coil embolization, using logistic regression analysis $(p=0.001)$. Asai et al. ${ }^{4)}$ also reported that the high intensity lesions on DWI were more frequently found in patients with HPR ( $39.4 \%$ vs. $21.2 \% ; p=0.01$ ), although no significant difference was found in symptomatic events between patients with and without HPR. Hwang et al. ${ }^{21)}$ prospectively evaluated the thromboembolic event rate in patients undergoing coil embolization and found that thromboembolic events more frequently occurred in patients with HPR than in those without HPR ( $11.1 \%$ vs. $1.0 \% ; p=0.03$ ).

Only a few studies have been carried out on bleeding events. Goh et al. ${ }^{17)}$ reported that LPR on clopidogrel therapy ( $\geq 72 \%$ inhibition) was associated with increased bleeding risk $(p=0.084)$. Kashiwazaki et al. ${ }^{23)}$ found that the $\geq 74 \%$ inhibition as the threshold for bleeding events could highly predict bleeding events. Nishi et al. ${ }^{33)}$ found that HPR (PRU $\left.\leq 175\right)$ had a 2.8 times higher risk for bleeding events in coiling and carotid stenting.

Several studies focusing on flow diversion also reported the association of platelet reactivity with thromboembolic and bleeding events. Daou et al. ${ }^{9)}$ revealed the higher rates of thromboembolic events in patients with PRU $>150$ and bleeding events in those with PRU $<70$ in flow diversion procedure. Delgado Almandoz et al. ${ }^{10)}$ reported that HPR (PRU >240) and LPR (PRU <60) on clopidogrel therapy led to increased numbers of thromboembolic and bleeding events in Pipeline placement, respectively. Tan et al. ${ }^{44)}$ divided clopidogrel response into two groups based on the 208 PRU value and evaluated thromboembolic events and high intensity lesions on DWI. While the overall thromboembolic events were more common in patients with PRU $>208$, no difference was observed in the high intensity lesions between groups with PRU $>208$ and $\leq 208^{44}$.

\section{PRU CUT-OFF VALUES FOR LPR, OPR, AND HPR IN NEUROINTERVENTION PROCEDURE}

The therapeutic window of platelet reactivity has been prospectively evaluated in numerous cardiology trials. A large meta-analysis of 17 studies including more than 20000 patients found an optimal range of platelet reactivity for the P2Y12 inhibitor, in which both clinical outcome and bleeding risk were lowest ${ }^{3}$. Current cardiology guideline defines LPR, OPR, and HPR categories as $<95,95-208$, and $>208$ PRU for the VerifyNow test (Accumetrics) ${ }^{3,18)}$.

In regard to target diseases and clinical events, neurointervention apparently differs with coronary intervention. Therefore, PRU cut-off values for neurointervention procedure 
should be based on its own data. Nevertheless, most neurointervention studies simply applied the cut-off values for coronary intervention without any validation. In a literature search, we found four neurontervention studies that were conducted to find the optimal cut-off value for predicting thromboembolic events ${ }^{17,22,23,25)}$. Two studies using the \% inhibition of the VerifyNow P2Y12 test (Accumetrics) were excluded in our review. Kang et al. ${ }^{22)}$ found $\geq 285$ PRU as a cut-off value for defining HPR on clopidogrel therapy in the coiling procedure. Although sensitivity and specificity are generally used to determine the best cut-off value in diagnostic test analysis, negative predictive value is important for interpreting the accuracy of the test in predicting thromboembolic events. While negative predictive value was not originally provided in their study, when calculated based on their reported data, it was $96.7 \%$. This indicates that thromboembolic events can occur in 3.3\% of patients who are identified as OPR using this cut-off value. They did not perform further validation study for this cut-off value, but reported a thromboembolic event rate of $6 \%$ in patients with OPR in their recent study using 285 PRU as the cut-off value ${ }^{8)}$.

Kim et al. ${ }^{25)}$ reported $\geq 220$ PRU as a cut-off value for defining HPR in a post-hoc analysis of their prospective data on coiling procedure. When using 220 PRU, the negative predictive value was $99.1 \%$ (thromboembolic risk of $0.9 \%$ in patients with OPR). They also estimated prediction accuracy for thromboembolic events using Bayes' theorem at various PRU cut-off values commonly used in most neurointervention studies. The estimated thromboembolic event rates were $1.0 \%, 1.0 \%, 0.9 \%, 3.2 \%$, and $3.2 \%$ at 208 (current cardiology guideline), 213, 220, 230, and 240 PRU (previous cardiology guideline). They also performed a prospective validation study and reported a thromboembolic event rate of $0.8 \%$ in patients with $<220$ PRU. In this review, we found that high PRU cutoff values of more than 220 PRU have considerable thromboembolic risk and low PRU cut-off values show unnecessary over-inhibition without additional benefit. Based on the currently available data, it is recommended that 220 PRU be selected as the cut-off value for categorizing platelet reactivity on P2Y12 inhibitor therapy in HPR and OPR.

The optimal cut-off value for predicting bleeding events in the neurointervention procedure was evaluated in four studies. Two studies using \% inhibition of VerifyNow P2Y12 test (Accumetrics) identified $\geq 72 \%$ and $74 \%$ inhibition as the cut- off value for $\mathrm{LPR}^{17,23)}$. Although these values showed good prediction accuracy (area under curve [AUC] in receiver-operation curve analysis, 0.96 for $\geq 72 \%$ inhibition and 0.82 for $\geq 74 \%$ inhibition), the use of $\%$ inhibition is no longer recommended. In a retrospective study, it was found that a PRU value of $\leq 175$ discriminated between patients with and without bleeding events $(19.0 \% \text { vs. } 7.7 \%)^{33)}$. However, because prediction accuracy of this value was graded as poor (AUC, 0.63), this cut-off value appears to be difficult to apply in practice. Kim et al. ${ }^{25)}$ conducted post-hoc analysis of a prospective trial and found that PRU value did not predict bleeding events. In this review, we therefore could not find optimal PRU cut-off value for predicting bleeding events in the neuintervention procedure. However, considering the potential bleeding risk, the use of a cardiology guideline (95 PRU) as the cut-off value for defining LPR is reasonable, although further studies are needed to address this issue $e^{3,18)}$.

\section{ANTIPLATELET THERAPY MODIFICATION BASED ON PLATELET FUNCTION TESTING}

A retrospective study evaluated the effect of high dose clopidogrel to overcome HPR in a neurointervention procedure ${ }^{14)}$. Tailoring clopidogrel dose based on platelet function testing was found to reduce thromboembolic event rate $(10.3 \%$ vs. $4.5 \%$ ), but this rate difference showed no statistical significance. Based on this study, a prospective trial was performed to evaluate the effect and safety of antiplatelet therapy modification for patients with HPR undergoing coil embolization for unruptured aneurysm ${ }^{21)}$. High dose $(300 \mathrm{mg}$ ) of aspirin was administrated in patients with HPR on aspirin therapy, and a loading dose $(200 \mathrm{mg}$ ) of cilostazol was added to standard dual antiplatelet therapy in patients with HPR on clopidogrel therapy in the morning of the procedure day. The antiplatelet therapy modification was found to significantly reduce thromboembolic event rate (11.1\% vs. $1.6 \%)$ without increasing bleeding risk.

Several small-sized case series found that prasugrel or ticagrelor could be used as an alternative to clopidogrel in patients with HPR on clopidogrel therapy undergoing various neurointervention procedures including coiling, flow diversion, and stenting ${ }^{1,7,20,43)}$. These studies reported high bleeding risk in patients receiving prasugrel, but no majority of bleed- 
ing occurred in the general coiling procedure, except for avoidable complications such as wire perforation and inaccurate femoral puncture. Prasugrel was also prospectively evaluated in coiling for intracranial aneurysm ${ }^{25}$. The loading dose (30 mg) of prasugrel was administrated for patients with HPR on clopidogrel therapy in the morning of the procedure day. Replacing clopidogrel with prasugrel in patients with HPR significantly reduced the thromboembolic event rate $(12.3 \%$ vs. $0.8 \%$ ), which was found to be similar to that $(0.8 \%)$ in patients with OPR on clopidogrel therapy. It was also found that prasugrel did not increase bleeding risk. Recent retrospective studies comparing prasugrel with clopidogrel in coil embolization also found that prasugrel provided consistent platelet inhibition and did not increase bleeding risk ${ }^{8,19)}$.

\section{CONCLUSION}

Current data on antiplatelet therapy in neurovascular intervention is insufficient. However, there is significant evidence for the prognostic value of the platelet function test and the effect of antiplatelet therapy modification based on test results. Among the various antiplatelet drugs, clopidogrel response variability is associated with clinical events, and thus platelet function testing for clopidogrel effect is needed before the neurointervention procedure. In particular, the VerifyNow P2Y12 test is useful in the evaluation of platelet reactivity on clopidogrel therapy and the modification of antiplatelet regimen, because of its ease of use and rapid feedback. The VerifyNow aspirin test (Accumetrics) can be omitted.

The suggested strategy for tailoring antiplatelet therapy is summarized in Fig. 1. Platelet function testing is recommended one day before the neurointervention procedure. Modified antiplatelet regimen can be effective, if it is administered at least 2 hours before the procedure (usually in the morning of the procedure day). For patients with HPR ( $\geq 220$ PRU), both adding cilostazol or replacing clopidogrel with prasugrel are effective. However, we recommend the use of prasugrel, because a high adverse event (headache) rate of cilostazol frequently leads to low compliance. For patients with LPR $(<95$ PRU), taking a half dose ( $37.5 \mathrm{mg}$ ) of clopidogrel every day is easier than taking one tablet $(75 \mathrm{mg})$ every other day. Current evidence does not support re-checking platelet reactivity nor delaying the procedure until platelet reactivity reaches OPR level (95-219 PRU). This guideline mainly targets coil embolization for intracranial aneurysm, because most available data were obtained from studies on coiling procedure. Further research is needed to develop guideline for antiplatelet therapy in stenting and flow diversion procedures.

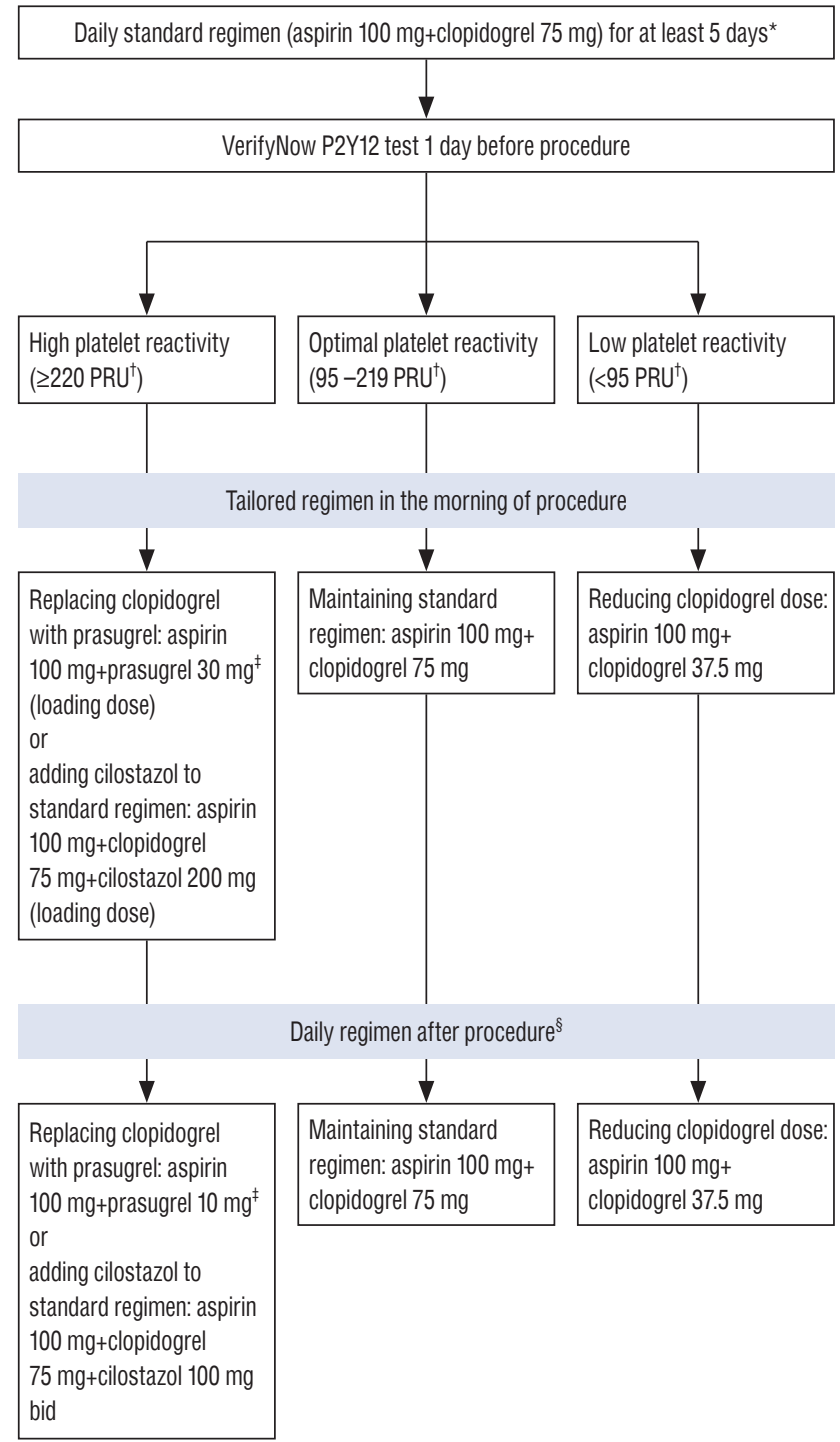

Fig. 1. Suggested strategy for tailoring antiplatelet therapy based on results of the VerifyNow P2Y12 test (Accumetrics, San Diego, CA, USA). *When loading doses of aspirin and clopidogrel are used, VerifyNow $\mathrm{P} 2 \mathrm{Y} 12$ is recommended 4 hours after administration. ${ }^{\dagger}$ For stenting for atherosclerotic stenosis or flow diversion, a cardiology guideline (208 PRU) may be selected for dividing platelet reactivity into high and optimal categories. " Loading dose of prasugrel is based on pharmacodynamic studies for Korean population ${ }^{26,31,46)}$. For patients with $<60 \mathrm{~kg}$ or $\geq 75$ years, prasugrel $20 \mathrm{mg}$ and $5 \mathrm{mg}$ are recommended as loading and maintenance doses, respectively. ${ }^{5}$ For patients receiving the procedure without stent placement, daily antiplatelet therapy is not generally recommended after procedure. PRU : P2Y12 reaction units. 


\section{CONFLICTS OF INTEREST}

No potential conflict of interest relevant to this article was reported.

\section{INFORMED CONSENT}

This type of study does not require informed consent.

\section{- Acknowledgements}

This review was supported by Hallym University Research Fund (HURF-2017-21).

\section{References}

1. Akbari SH, Reynolds MR, Kadkhodayan Y, Cross DT 3rd, Moran CJ : Hemorrhagic complications after prasugrel (Effient) therapy for vascular neurointerventional procedures. J Neurointerv Surg 5 : 337-343, 2013

2. Aradi D, Collet JP, Mair J, Plebani M, Merkely B, Jaffe AS, et al. : Platelet function testing in acute cardiac care - is there a role for prediction or prevention of stent thrombosis and bleeding? Thromb Haemost 113 : 221-230, 2015

3. Aradi D, Kirtane A, Bonello L, Gurbel PA, Tantry US, Huber K, et al. : Bleeding and stent thrombosis on P2Y12-inhibitors: collaborative analysis on the role of platelet reactivity for risk stratification after percutaneous coronary intervention. Eur Heart J 36 : 1762-1771, 2015

4. Asai T, Miyachi S, Izumi T, Matsubara N, Haraguchi K, Yamanouchi T, et al. : Relationship between low response to clopidogrel and periprocedural ischemic events with coil embolization for intracranial aneurysms. J Neurointerv Surg 8 : 752-755, 2016

5. Buch AN, Singh S, Roy P, Javaid A, Smith KA, George CE, et al. : Measuring aspirin resistance, clopidogrel responsiveness, and postprocedural markers of myonecrosis in patients undergoing percutaneous coronary intervention. Am J Cardiol 99 : 1518-1522, 2007

6. Cattaneo M : Laboratory detection of 'aspirin resistance': what test should we use (if any)? Eur Heart J 28 : 1673-1675, 2007

7. Chalouhi N, Tjoumakaris S, Dumont AS, Gonzalez LF, Randazzo C, Starke RM, et al. : Treatment of posterior circulation aneurysms with the pipeline embolization device. Neurosurgery 72 : 883-889, 2013

8. Choi HH, Lee JJ, Cho YD, Han MH, Cho WS, Kim JE, et al. : Antiplatelet premedication for stent-assisted coil embolization of intracranial aneurysms: low-dose prasugrel vs clopidogrel. Neurosurgery 83 : 981 988, 2018

9. Daou B, Starke RM, Chalouhi N, Barros G, Tjoumakaris S, Rosenwasser RH, et al. : P2Y12 reaction units: effect on hemorrhagic and thromboem- bolic complications in patients with cerebral aneurysms treated with the pipeline embolization device. Neurosurgery 78 : 27-33, 2016

10. Delgado Almandoz JE, Crandall BM, Scholz JM, Fease JL, Anderson RE, Kadkhodayan $\mathrm{Y}$, et al. : Pre-procedure P2Y12 reaction units value predicts perioperative thromboembolic and hemorrhagic complications in patients with cerebral aneurysms treated with the pipeline embolization device. J Neurointerv Surg 5 Suppl 3 : iii3-iii10, 2013

11. Douketis JD, Berger PB, Dunn AS, Jaffer AK, Spyropoulos AC, Becker $\mathrm{RC}$, et al. : The perioperative management of antithrombotic therapy: American College of Chest Physicians Evidence-Based Clinical Practice Guidelines (8th edition). Chest 133 (6 Suppl) : 299S-339S, 2008

12. Drazin D, Choulakian A, Nuño M, Kornbluth P, Alexander MJ : Body weight: a risk factor for subtherapeutic antithrombotic therapy in neurovascular stenting. J Neurointerv Surg 3 : 177-181, 2011

13. Farid NA, Payne CD, Small DS, Winters KJ, Ernest CS 2nd, Brandt JT, et al. : Cytochrome P450 3A inhibition by ketoconazole affects prasugrel and clopidogrel pharmacokinetics and pharmacodynamics differently. Clin Pharmacol Ther 81 : 735-741, 2007

14. Fifi JT, Brockington C, Narang J, Leesch W, Ewing SL, Bennet $H$, et al. : Clopidogrel resistance is associated with thromboembolic complications in patients undergoing neurovascular stenting. AJNR Am J Neuroradiol 34 : 716-720, 2013

15. Gaglia MA, Torguson R, Pakala R, Xue Z, Sardi G, Suddath WO, et al. : Correlation between light transmission aggregometry, VerifyNow P2Y12, and VASP-P platelet reactivity assays following percutaneous coronary intervention. J Interv Cardiol 24 : 529-534, 2011

16. Godino C, Mendolicchio L, Figini F, Latib A, Sharp AS, Cosgrave J, et al. : Comparison of VerifyNow-P2Y12 test and flow cytometry for monitoring individual platelet response to clopidogrel. What is the cut-off value for identifying patients who are low responders to clopidogrel therapy? Thromb J $7:$ 4, 2009

17. Goh C, Churilov L, Mitchell P, Dowling R, Yan B : Clopidogrel hyperresponse and bleeding risk in neurointerventional procedures. AJNR Am J Neuroradiol 34 : 721-726, 2013

18. Gross $L$, Aradi $D$, Sibbing $D$ : Platelet function testing in patients on antiplatelet medications. Semin Thromb Hemost 42 : 306-320, 2016

19. Ha EJ, Cho WS, Kim JE, Cho YD, Choi HH, Kim T, et al. : Prophylactic antiplatelet medication in endovascular treatment of intracranial aneurysms: low-dose prasugrel versus clopidogrel. AJNR Am J Neuroradiol 37 : 2060-2065, 2016

20. Hanel RA, Taussky P, Dixon T, Miller DA, Sapin M, Nordeen JD, et al. : Safety and efficacy of ticagrelor for neuroendovascular procedures. A single center initial experience. J Neurointerv Surg 6 : 320-322, 2014

21. Hwang G, Huh W, Lee JS, Villavicencio JB, Villamor RB Jr, Ahn SY, et al. : Standard vs modified antiplatelet preparation for preventing thromboembolic events in patients with high on-treatment platelet reactivity undergoing coil embolization for an unruptured intracranial aneurysm: a randomized clinical trial. JAMA Neurol 72 : 764-772, 2015

22. Kang HS, Kwon BJ, Kim JE, Han MH : Preinterventional clopidogrel response variability for coil embolization of intracranial aneurysms: clinical implications. AJNR Am J Neuroradiol 31 : 1206-1210, 2010 
23. Kashiwazaki D, Kuwayama N, Akioka N, Hayakawa Y, Kuroda S : The roles and issues of P2Y12 percent inhibition assessed by VerifyNow assay for patients undergoing Neurointervention: a prospective study. J Stroke Cerebrovasc Dis 23 : 1830-1836, 2014

24. Kim B, Kim K, Jeon P, Kim S, Kim H, Byun H, et al. : Thromboembolic complications in patients with clopidogrel resistance after coil embolization for unruptured intracranial aneurysms. AJNR Am J Neuroradiol 35 : 1786-1792, 2014

25. Kim CH, Hwang G, Kwon OK, Ban SP, Chinh ND, Tjahjadi M, et al. : $\mathrm{P} 2 \mathrm{Y} 12$ reaction units threshold for implementing modified antiplatelet preparation in coil embolization of unruptured aneurysms: a prospective validation study. Radiology 282 : 542-551, 2017

26. Kim MH, Zhang $\mathrm{HZ}$, Jung DK : Pharmacodynamic comparisons for single loading doses of prasugrel $(30 \mathrm{mg})$ and clopidogrel $(600 \mathrm{mg})$ in healthy Korean volunteers. Circ J 77 : 1253-1259, 2013

27. Koerner H, Derveaux C, Alexandrou M, Graeber S, Roth C, Papanagiotou $P$, et al. : Do clopidogrel nonresponders have an increased risk of adverse events during supra-aortal angioplasty and stenting? Stroke Res Treat 2012 : 904534, 2012

28. Kovács EG, Katona É, Bereczky Z, Homoródi N, Balogh L, Tóth E, et al. : Evaluation of laboratory methods routinely used to detect the effect of aspirin against new reference methods. Thromb Res 133 : 811-816, 2014

29. Kovács EG, Katona É, Bereczky Z, Homoródi N, Balogh L, Tóth E, et al. : New direct and indirect methods for the detection of cyclooxygenase 1 acetylation by aspirin; the lack of aspirin resistance among healthy individuals. Thromb Res 131 : 320-324, 2013

30. Lee DH, Arat A, Morsi H, Shaltoni H, Harris JR, Mawad ME : Dual antiplatelet therapy monitoring for neurointerventional procedures using a point-of-care platelet function test: a single-center experience. AJNR Am J Neuroradiol 29 : 1389-1394, 2008

31. Lee DH, Kim MH, Guo LZ, Park MK, Yi SJ : Lower loading dose of prasugrel compared with conventional loading doses of clopidogrel and prasugrel in Korean patients undergoing elective coronary angiography: a randomized controlled study evaluating pharmacodynamic efficacy. Korean Circ J 44 : 386-393, 2014

32. Michelson AD, Cattaneo M, Eikelboom JW, Gurbel P, Kottke-Marchant $\mathrm{K}$, Kunicki TJ, et al. : Aspirin resistance: position paper of the working group on aspirin resistance. J Thromb Haemost 3 : 1309-1311, 2005

33. Nishi H, Nakahara I, Matsumoto S, Hashimoto T, Ohta T, Sadamasa N, et al. : Platelet reactivity and hemorrhage risk in neurointerventional procedures under dual antiplatelet therapy. J Neurointerv Surg 8 : 949-953, 2016

34. Nordeen JD, Patel AV, Darracott RM, Johns GS, Taussky P, Tawk RG, et al. : Clopidogrel resistance by P2Y12 platelet function testing in patients undergoing neuroendovascular procedures: incidence of ischemic and hemorrhagic complications. J Vasc Interv Neurol 6 : 26-34, 2013

35. Pandya DJ, Fitzsimmons BF, Wolfe TJ, Hussain SI, Lynch JR, OrtegaGutierrez S, et al. : Measurement of antiplatelet inhibition during neurointerventional procedures: the effect of antithrombotic duration and loading dose. J Neuroimaging 20 : 64-69, 2010

36. Paniccia R, Priora R, Liotta AA, Abbate R : Platelet function tests: a comparative review. Vasc Health Risk Manag 11 : 133-148, 2015

37. Paré G, Mehta SR, Yusuf S, Anand SS, Connolly SJ, Hirsh J, et al. : Effects of CYP2C19 genotype on outcomes of clopidogrel treatment. $\mathbf{N}$ Engl J Med 363 : 1704-1714, 2010

38. Patrono C, García Rodríguez LA, Landolfi R, Baigent C : Low-dose aspirin for the prevention of atherothrombosis. N Engl J Med 353 : 23732383, 2005

39. Pierot L, Wakhloo AK : Endovascular treatment of intracranial aneurysms: current status. Stroke 44 : 2046-2054, 2013

40. Prabhakaran S, Wells KR, Lee VH, Flaherty CA, Lopes DK : Prevalence and risk factors for aspirin and clopidogrel resistance in cerebrovascular stenting. AJNR Am J Neuroradiol 29 : 281-285, 2008

41. Scott SA, Sangkuhl K, Shuldiner AR, Hulot JS, Thorn CF, Altman RB, et al. : PharmGKB summary: very important pharmacogene information for cytochrome P450, family 2, subfamily C, polypeptide 19. Pharmacogenet Genomics 22 : 159-165, 2012

42. Simon T, Steg PG, Gilard M, Blanchard D, Bonello L, Hanssen M, et al. : Clinical events as a function of proton pump inhibitor use, clopidogrel use, and cytochrome P450 2C19 genotype in a large nationwide cohort of acute myocardial infarction: results from the French Registry of Acute ST-Elevation and Non-ST-Elevation Myocardial Infarction (FAST-MI) registry. Circulation $123:$ 474-482, 2011

43. Stetler WR, Chaudhary N, Thompson BG, Gemmete JJ, Maher CO, Pandey AS : Prasugrel is effective and safe for neurointerventional procedures. J Neurointerv Surg 5 : 332-336, 2013

44. Tan LA, Keigher KM, Munich SA, Moftakhar R, Lopes DK : Thromboembolic complications with pipeline embolization device placement: impact of procedure time, number of stents and pre-procedure P2Y12 reaction unit (PRU) value. J Neurointerv Surg 7 : 217-221, 2015

45. Tantry US, Bonello L, Aradi D, Price MJ, Jeong YH, Angiolillo DJ, et al. : Consensus and update on the definition of on-treatment platelet reactivity to adenosine diphosphate associated with ischemia and bleeding. J Am Coll Cardiol 62 : 2261-2273, 2013

46. Yu KS, Park KW, Kelly RP, Gu N, Payne C, Small DS, et al. : Pharmacokinetic and pharmacodynamic effects of prasugrel in healthy Korean males. J Cardiovasc Pharmacol 62 : 72-77, 2013 